\title{
Thickness, and a categoric view of type-space functors
}

\author{
by
}

\author{
Itay Ben-Yaacov (Cambridge, MA)
}

\begin{abstract}
We define the class of thick cats (compact abstract theories, which contains in particular semi-Hausdorff, Hausdorff and first order cats), and prove that in this class simplicity behaves as in first order theories. We consider well-known first order notions, such as interpretability or stable dividing/reduct, and propose analogous notions that can be naturally expressed in terms of maps between type-space functors. We prove several desirable properties of the new notions and show the connection between them and their classical counterparts. We conclude with several scattered results concerning cats and simplicity.
\end{abstract}

Introduction. In [Ben03] we defined cats (or compact abstract theories), which may be viewed as a model-theoretic framework with compactness but without negation. It is more general than the first order framework, and can accommodate, for example, various kinds of analytic structures that do not admit a first order description. In fact, we showed the equivalence of several quite different approaches to the definition of cats, each having its own merits.

The first and most concrete presentation of a cat is via a particular kind of universal domains which are homogeneous and compact in a language without negation. Types, dividing, etc., are defined more or less as usual inside a universal domain. In [Benb] we showed how simplicity can be developed in this context from the assumption that non-dividing has the local character, even though this does not imply that non-dividing extensions always exist.

In the first section of the present paper we introduce the class of thick cats, i.e., cats where indiscernibility is type-definable. We show that the thickness assumption is very mild, and that with this assumption many

2000 Mathematics Subject Classification: 03C95, 03C45.

Key words and phrases: cats, simplicity, thickness.

At the time of the writing of this paper, the author was a graduate student with the Équipe de Logique Mathématique of Université Paris VII. 
properties of first order simplicity which do not necessarily hold in a general simple cat, do hold in a simple thick cat:

(i) If $p \in \mathrm{S}(A)$ and $B \supseteq A$ then $p$ has non-dividing extensions to $B$.

(ii) Moreover, with the same assumptions, the set $\left\{a: a \vDash p \wedge a \downarrow_{A} B\right\}$ is type-definable (we would say that complete types have definable independence).

(iii) The theory of definable groups (which corresponds to the theory of hyperdefinable groups in first order theories) can be developed as well. We content ourselves with showing that generic types exist.

(iv) As a side remark, even though the results in [Ben02] (and in its sequels, [BTW, BW]) are stated for first order theories, a closer inspection would reveal that one only uses the existence of non-dividing extensions, definable independence for complete types and the fact that indiscernibility is type-definable. In other words, the theorems contained therein are about simple thick cats.

The second section uses the fact that a cat is essentially characterised by its type-spaces, or more precisely by its type-space functor. This section stems from the intuitive feeling that relations between two theories are most elegantly viewed, and in fact should be viewed, as mappings between their type-spaces. We consider two such relations, namely that of interpretation of one theory in another and that of a stable reduct of a simple theory, and try to present them in this manner. We obtain the notions of description and stable representations, respectively, which are more general than the original ones, and allow us to strengthen some known results. For example:

(i) A simple thick cat is unstable if and only if it describes the random graph.

(ii) If a simple theory has a stable representation then it has stable dividing, and all known simple theories have stable representations.

In $[\mathrm{BPV}, \mathrm{Bena}]$ we define the theory $T^{P}$ of lovely pairs of models of a simple first order theory, or more generally of a thick cat $T$. We show in particular that $T^{P}$ admits a natural description in $T$ which also preserves independence. It follows quite easily that if $T$ admits a stable representation then so does $T^{P}$. Such a preservation result is not at all clear for, say, stable dividing.

The third section is a collection of various results, mostly independent of each other and of the first two sections, which the reader is invited to browse at her or his ease.

Let us give a few reminders concerning cats.

Definition 0.1. Let $\mathcal{L}$ be a first order language, and fix a positive fragment of $\mathcal{L}$, i.e., a subset $\Delta \subseteq \mathcal{L}$ which is closed under positive boolean 
combinations (actually, $\mathcal{L}$ is completely unimportant, all we want is $\Delta$ ). A formula, unless otherwise qualified, is always a member of $\Delta$, and similarly for partial types.

A universal domain (with respect to $\Delta$ ) is a structure $U$ satisfying:

(i) Strong homogeneity: If $A, B \subseteq U$ are small and $f: A \rightarrow B$ is a $\Delta$-homomorphism (i.e., for any $\varphi \in \Delta$ and $a \in A, U \vDash \varphi(a) \Rightarrow U \vDash$ $\varphi(f(a))$ ), then $f$ extends to an automorphism of $U$ (so in particular, $f$ is a $\Delta$-isomorphism of $A$ and $B$ ).

(ii) Compactness: Every small partial $\Delta$-type over $U$ which is finitely realised in $U$ is realised in $U$.

Although this is not required by the definition, we will also assume that every existential formula, i.e., formula of the form $\exists y \varphi(x, y)$ where $\varphi \in \Delta$, is equivalent in $U$ to a partial $\Delta$-type. (If not, we can always close $\Delta$ under existential quantification without harming either compactness or homogeneity; this is just usually unnecessary.)

Saturated and strongly homogeneous models of first order theories are one example of universal domain (with $\Delta=\mathcal{L}$ ). Another easy example which we will refer to later on is that of Hilbert spaces:

FACT 0.2. Let $H$ be the unit ball of a very large Hilbert space. Let $\Delta$ be the set of all formulas of the form $s \leq\left\|\sum_{i<n} \lambda_{i} x_{i}\right\| \leq r$ (closed under positive boolean combinations). Then $H$ is a universal domain with respect to $\Delta$.

The negative universal theory of a universal domain

$$
\operatorname{Th}_{\Pi}(U)=\{\forall \bar{x} \neg \varphi: \varphi(\bar{x}) \in \Delta, U \vDash \forall \bar{x} \neg \varphi(\bar{x})\}
$$

has the property that the category of subsets of its e.c. models has the amalgamation property (there is a little twist here, since the notion of e.c. models is defined with respect to $\Delta$-homomorphisms). A negative universal theory having this property is called a positive Robinson theory. Conversely, if $T$ is a positive Robinson theory, and in addition is complete (i.e., the category of its e.c. models has the joint embedding property), then $T=\mathrm{Th}_{\Pi}(U)$ for some universal domain $U$. Thus the giving of a universal domain is essentially the same as the giving of a positive Robinson theory. Henceforth, a theory means a positive Robinson theory, unless explicitly stated otherwise.

To a universal domain $U$, or to a theory $T$, we associate type-spaces: for every set of indices $I$ we define $\mathrm{S}_{I}(T)$ as the set of all maximal types in $\alpha$ variables which are consistent with $T$. If $U$ is a universal domain for $T$ then this is the same as $U^{I} / \operatorname{Aut}(U)$, by homogeneity. We put a compact and $T_{1}$ topology on $\mathrm{S}_{I}(T)$ by taking the closed sets to be those defined by partial types. If $\mathrm{S}_{n}(T)$ is Hausdorff for every $n<\omega$ then $\mathrm{S}_{I}(T)$ is Hausdorff for every 
set $I$, and we say that $T$ is Hausdorff. One consequence of being Hausdorff is that the property of two tuples to have the same type is a type-definable property. If only the latter holds, we say that $T$ is semi-Hausdorff.

1. Thick cats. We adapt the notion of a thick formula, originally defined by Ziegler, to the context of cats. We then define a thick cat as one which has enough thick formulas to define indiscernibility by a partial type, and prove that simplicity theory for thick cats is a rather complete generalisation of the first order case, and does not suffer from the many deficiencies of simplicity theory for arbitrary cats.

The class of thick cats is a very large one: it contains all semi-Hausdorff cats, and therefore all Hausdorff and first order cats, which means almost all known examples. On the other hand, we show in [Benb, Example 4.3] that ultrametric spaces with distances in $\omega$ form a stable cat where not all types have non-dividing extensions; it follows from what we show below that this cat cannot be thick.

In order to be able to take advantage of thickness, we introduce the total $D$-rank, which calculates at once all local $D$-ranks. This rank is witnessed by a tree, or by a sequence of dividing formulas, which are not well-founded (the order type of the sequence or of the levels of the tree is an inverse ordinal). This may seem unnatural at first, and poses technical problems when one wants to use proof by induction (essentially for Proposition 1.12). Instead, we use induction for the finite case, where an inverse ordinal is also well-ordered, and then use compactness and thickness to extend the result to the infinite case. We conclude that the local character of dividing gives the same theory of independence as in the first order case (plugging in results from [Benb], or just following the development in [Pil00]).

Thickness is also used in order to show that the set of complete types over a set $A$ that have a given (or higher) $D$-rank is closed: in the first order case we would just take the negations of all formulas that would make the $D$-rank too low, but this cannot be done in a cat. This application of thickness is essential for the proof that every definable group has generic elements, as we show by a counterexample.

1.1. Thickness. Thick formulas were defined by Ziegler in the context of first order theories; for example, see [CLPZ01, Definition 1.10]. However, this definition is not suitable in the context of cats, so we use one of the equivalent conditions of [CLPZ01, Fact 1.11] instead. Thin formulas are defined accordingly.

Definition 1.1. Let $\varphi(x, y)$ be a formula where $x$ and $y$ are of the same sort (that is, tuples of variables of the same length and sorts). 
(i) $\varphi(x, y)$ is thin if for no indiscernible sequence $\left(a_{i}: i<\omega\right)$ do we have $\varphi\left(a_{0}, a_{1}\right)$.

(ii) $\varphi(x, y)$ is thick if $\varphi\left(a_{0}, a_{1}\right)$ holds for every such indiscernible sequence.

This should be obvious:

FACT 1.2. (i) $\varphi(x, y)$ is thin if and only if there is $n<\omega$ such that there is no sequence $\left(a_{i}: i<n\right)$ with $\varphi\left(a_{i}, a_{j}\right)$ for every $i<j<n$. If $n$ is the minimal such, we say that $\varphi$ is $n$-thin.

(ii) $\varphi(x, y)$ is thick if and only if all its negations are thin.

We see that if $\varphi$ has a thick negation, then it is thin. The converse of this observation would be that if $\varphi$ is thin then it has a thick negation. This would mean, in a sense, that $T$ "has enough thick formulas":

Definition 1.3. A cat $T$ is thick if every thin formula has a thick negation.

Definition 1.4. Let $a$ and $b$ be two tuples of the same length (finite or infinite), and $A$ a set. Then $d_{A}(a, b)$ is the minimal $n<\omega$ such that there are $a=a_{0}, \ldots, a_{n}=b$ and $a_{i}, a_{i+1}$ can be continued to an $A$-indiscernible sequence for all $i<n$. If no such $n$ exists then $d_{A}(a, b)=\infty$. We write $d$ instead of $d_{\emptyset}$.

Proposition 1.5. The following are equivalent:

(i) $T$ is thick.

(ii) Every 3-thin formula has a thick negation.

(iii) The property that an infinite sequence is indiscernible is typedefinable.

(iv) The property $d(x, y) \leq 1$ is type-definable.

(v) The property $d_{A}(x, y) \leq 1$ is type-definable for every set $A$.

Proof. (i) $\Rightarrow$ (ii). Clear.

(ii) $\Rightarrow$ (iii). For two tuples of variables of the same sort $x$ and $y$, define $\pi(x, y)$ as the set of all thick formulas in $x$ and $y$ (it varies, of course, with the sort of $x$ and $y$, but since the sort is always clear from the context we just write $\pi$ ).

If $a$ and $b$ lie on some indiscernible sequence, then necessarily $\pi(a, b)$, since $\pi$ only contains thick formulas. On the other hand, if $a \not \equiv b$, then there are contradictory formulas $\varphi(x)$ and $\psi(x)$ such that $\vDash \varphi(a) \wedge \psi(b)$; the formula $\chi(x, y)=\varphi(x) \wedge \psi(y)$ is then 3-thin, so by assumption $\pi$ contradicts it. This shows that

$$
d(a, b) \leq 1 \Rightarrow \vDash \pi(a, b) \Rightarrow a \equiv b .
$$


Given a sequence of (tuples of) variables $\left(x_{i}: i<\omega\right)$, we define

$$
\Theta_{\text {ind }}(\bar{x})=\bigwedge_{n<\omega ; i_{0}<\ldots<i_{n-1}<j_{0}<\ldots<j_{n-1}} \pi\left(x_{i_{0}} \ldots x_{i_{n-1}}, x_{j_{0}} \ldots x_{j_{n-1}}\right) .
$$

One verifies that $\Theta_{\text {ind }}$ defines the property of being an indiscernible sequence. (iii) $\Rightarrow$ (iv). $d(x, y) \leq 1$ is defined by $\exists z_{<\omega} \Theta_{\text {ind }}\left(x, y, z_{<\omega}\right)$, where $\Theta_{\text {ind }}$ defines the property of being an indiscernible sequence (existential quantification of a partial type is equivalent to a partial type, by compactness).

(iv) $\Rightarrow($ i). The partial type defining $d(x, y) \leq 1$ can only contain thick formulas, and in fact it is equivalent to the set of all thick formulas. If $\varphi(x, y)$ is thin then it contradicts $d(x, y) \leq 1$, and therefore it contradicts some thick formula.

(iv) $\Leftrightarrow(\mathrm{v}) . \quad d_{A}(x, y) \leq 1$ is just $d(x A, y A) \leq 1 . \mathbf{m}_{1.5}$

We recall that a cat is said to be Hausdorff if its type-spaces are, and it is semi-Hausdorff if equality of types is type-definable. Then Hausdorff implies semi-Hausdorff, and semi-Hausdorff implies thick.

1.2. Simplicity. We aim to show that in a thick cat, simplicity behaves much like in the first order case: the local character of ordinary dividing implies the extension axiom.

Convention 1.6. We work in a thick cat $T$.

The reason that extension does not follow in cats from the local character by classical arguments is that a type over $A$ does not have a non-dividing extension to $B$ if and only if it implies an infinite disjunction $\bigvee_{i<\lambda} \varphi_{i}\left(x, b_{i}\right)$ where each $\varphi_{i}\left(x, b_{i}\right)$ is a formula over $B$ which divides over $A$, and we do not know whether this can be reduced to a finite disjunction. Therefore, we need a rank that can handle infinitely many formulas at a time.

We define a rank which calculates in one blow all local $D$-ranks, and a bit more. Recall first that if $\varphi(x, y)$ and $\psi\left(y_{<k}\right)$ are formulas, then $\psi$ is a $k$-inconsistency witness for $\varphi$ if $\psi\left(y_{<k}\right) \wedge \bigwedge_{i<k} \varphi\left(x, y_{i}\right)$ is contradictory; and in this case, $\varphi(x, b)$ divides over $c$ with respect to $\psi$ if there is a $c$-indiscernible sequence $\left(b_{i}: i<\omega\right)$ such that $b_{0}=b$ and $\vDash \psi\left(b_{0}, \ldots, b_{k-1}\right)$.

Notation 1.7. Let us fix a sort by naming a variable $x$ in that sort. $\Xi(x)$ is the set of all pairs $(\varphi, \psi)$, where $\varphi(x, y)$ is any formula ( $x$ is fixed, but $y$ may vary) and $\psi\left(y_{<k}\right)$ is a $k$-inconsistency witness for $\varphi$, for some $k<\omega$.

$\Xi(x)^{*}$ is the class of all sequences of ordinal length in $\Xi(x)$, and if $\bar{\xi} \in$ $\Xi(x)^{\alpha}$ we also write it as $\xi_{<\alpha}=\left(\xi_{i}: i<\alpha\right)=\left(\left(\varphi_{i}, \psi_{i}\right): i<\alpha\right)$, where each $\psi_{i}$ is a $k_{i}$-inconsistency witness for $\varphi_{i}\left(x, y_{i}\right)$.

If $\bar{\xi}, \bar{\zeta} \in \Xi(x)^{*}$ then $\bar{\xi} \leq \bar{\zeta}$ if $\bar{\xi}$ is an initial segment of $\bar{\zeta}$.

Ordinarily $x$ is clear from the context, so we just write $\Xi$. 
Definition 1.8. Let $p(x)$ be a partial type, say over $A$. Its total $D$-rank, denoted $D(p, \Xi)$, is a subclass of $\Xi^{*}$. For $\xi_{<\alpha} \in \Xi^{\alpha}$, we define whether $\xi_{<\alpha} \in D(p, \Xi)$ by induction on $\alpha$ :

- $\alpha=0: \emptyset \in D(p, \Xi)$ if $p$ is consistent.

- $\alpha$ limit: $\xi_{<\alpha} \in D(p, \Xi)$ if $\xi_{<\beta} \in D(p, \Xi)$ for every $\beta<\alpha$.

- $\alpha=\beta+1$ : Write $\xi_{\beta}=(\varphi, \psi)$. Then $\xi_{<\alpha} \in D(p, \Xi)$ if there exists $b$ such that $\varphi(x, b)$ divides over $A$ with respect to $\psi$, and $\xi_{<\beta} \in D(p \wedge \varphi(x, b), \Xi)$.

Remark 1.9. If $A \subseteq B$ and $\varphi(x, b)$ divides over $A$ with respect to $\psi$, then there is $b^{\prime} \equiv_{A} b$ such that $\varphi\left(x, b^{\prime}\right)$ divides over $B$ with respect to $\psi$. Thus the precise choice of the set of parameters $A$ is not important.

We could have just as well defined $D(-, \Omega)$ for any $\Omega \subseteq \Xi$. Then the new rank is a natural extension of the old local ones, since $D(p,\{(\varphi, \psi)\})$ is essentially the same thing as the rank $D(p, \varphi, \psi)$ (that is, $D(p, \varphi, \psi, 1)$ ) from $[$ Benb].

Definition 1.10. Let $A$ be some set, and $\bar{\xi}=\left(\left(\varphi_{i}, \psi_{i}\right): i<\alpha\right) \in \Xi^{\alpha}$. We say that $a$ satisfies $\operatorname{div}_{\bar{\xi}, A}$ if there exists a sequence $\left(b_{i}: i<\alpha\right)$ such that $\varphi_{i}\left(x, b_{i}\right)$ divides over $A \cup b_{>i}$ with respect to $\psi_{i}$ for every $i<\omega$ and $\vDash \bigwedge_{i} \varphi_{i}\left(a, b_{i}\right)$.

Lemma 1.11. $\operatorname{div}_{\bar{\xi}, A}(x)$ is defined by a partial type over $A$.

Proof. First, for every pair $\left(\varphi(x, y), \psi\left(y_{<k}\right)\right) \in \Xi$, there is a partial type $p_{\varphi, \psi, A}(y)$ over $A$ such that $\vDash p_{\varphi, \psi, A}(b)$ if and only if $\varphi(x, b)$ divides over $A$ with respect to $\psi$ : all we have to say is that there exist $\left(y_{j}: j<\omega\right)$ which are $A$-indiscernible (this is where thickness comes into play) satisfying $\psi\left(y_{<k}\right) \wedge$ $y=y_{0}$.

Now $\operatorname{div}_{\bar{\xi}, A}(x)$ can be expressed by saying that there exist $\left(y_{i}: i<\alpha\right)$ such that $\varphi_{i}\left(x, y_{i}\right) \wedge p_{\varphi_{i}, \psi_{i}, A y_{>i}}\left(y_{i}\right)$ holds for each $i<\alpha \cdot \mathbf{- 1}_{1.11}$

The fundamental property of the $D(-, \Xi)$ rank is the following:

Proposition 1.12. Let $p(x)$ be a partial type over a set $A$ and $\bar{\xi} \in \Xi^{\alpha}$. Then $\bar{\xi} \in D(p, \Xi)$ if and only if $\operatorname{div}_{\bar{\xi}, A}(x) \wedge p(x)$ is consistent.

Proof. Right to left is by induction on $\alpha: 0$ and limit are trivial. For $\alpha=\beta+1$, let $a$ realise $\operatorname{div}_{\bar{\xi}, A}(x) \wedge p(x)$, as witnessed by a sequence $b_{<\alpha}$. Then $b_{<\beta}$ witness that $\operatorname{div}_{\xi_{<\beta}, A b_{\beta}}(a) \wedge p(a) \wedge \varphi_{\beta}\left(a, b_{\beta}\right)$, so $\xi_{<\beta} \in D\left(p \wedge \varphi_{\beta}\left(x, b_{\beta}\right), \Xi\right)$ by the induction hypothesis. Since in addition $\varphi_{\beta}\left(x, b_{\beta}\right)$ divides over $A$ with respect to $\psi_{\beta}$, we get $\xi_{<\alpha} \in D(p, \Xi)$.

Left to right: For $\alpha=0$ this is trivial. For $\alpha$ successor, the mirror image of the argument above works. For $\alpha$ limit observe that $D(p, \Xi)$ is closed for subsequences and apply the type-definability of $\operatorname{div}_{\bar{\xi}, A}$, the finite case, and 
compactness. (This last argument works in fact for every infinite $\alpha$, not only limit.) $\mathbf{- 1 . 1 2}$

Corollary 1.13. (i) Assume that $p$ is over $A$ and $\bar{\xi} \in D(p, \Xi)$. Then there exists $q \in \mathrm{S}(A)$ extending $p$ with $\bar{\xi} \in D(q, \Xi)$.

(ii) If $p(x, Y)$ is a partial type over $B$, then the set $\{A: \bar{\xi} \in D(p(x, A), \Xi)\}$ is type-definable by a partial type in $Y$ over $B$.

(iii) The property $\bar{\xi} \in D(x / Y, \Xi)$ is type-definable by a partial type in $x, Y$.

(iv) $\bar{\xi} \in D(p, \Xi)$ if and only if every finite subsequence of $\bar{\xi}$ belongs to $D(p, \Xi)$.

Proof. (i) Let $a$ realise $p \wedge \operatorname{div}_{\bar{\xi}, A}$. Then $\bar{\xi} \in D(a / A, \Xi)$.

(ii) We can express this as: $\exists x \operatorname{div}_{\bar{\xi}, Y B}(x) \wedge p(x, Y)$.

(iii) This is just $\operatorname{div}_{\bar{\xi}, Y}(x)$.

(iv) By compactness. 1.13

REMARK 1.14. Note that in the definition of $\operatorname{div}_{\bar{\xi}, A}(x)$, each formula is expected to divide over the tail of the sequence, and not over its head. Thus, the property $\xi_{<\alpha} \in D(p, \Xi)$ is equivalent to the existence of a corresponding tree, the order type of whose levels is $\alpha^{*}$, that is, $\alpha$ with inverse order. In particular, if $\alpha$ is infinite, the tree is not well-founded, and its definition is rather cumbersome. This is why we skip the definition of the corresponding tree, and use $\operatorname{div}_{\bar{\xi}, A}$ instead. Thickness is used to get around the fact that $\alpha^{*}$ is not well-ordered.

Of course, the same is true for ordinary local $D$-rank, which is just a special case of the total $D$-rank, but since ranks are then finite, we usually consider the sequence (or tree) in the "wrong" direction without noticing.

We have all the tools necessary to prove:

THEOREM 1.15. In a thick simple cat, every complete type has nondividing extensions to every set.

Proof. Let $p \in \mathrm{S}(A)$, and $B \supseteq A$. Since $T$ is assumed to be simple, $D(p, \Xi)$ is a set: in fact, $D(p, \Xi) \subseteq \Xi^{<|T|^{+}}$. Thus by Zorn's lemma, there is a maximal $\bar{\xi} \in D(p, \Xi)$. By Corollary 1.13 we can find $q \in \mathrm{S}(B)$ extending $p$, with $\bar{\xi} \in D(q, \Xi)$. Assume that $q$ divides over $A$, say $q \vdash \varphi(x, b)$ and $\varphi(x, b)$ divides over $A$ with respect to $\psi$. Then $(\bar{\xi},(\varphi, \psi)) \in D(p, \Xi)$, contradicting the maximality of $\bar{\xi} \cdot \mathbf{- 1 . 1 5}$

Here are a few useful observations. We assume that $T$ is thick and simple.

Proposition 1.16. (i) Equality of Lascar strong types over $A$ is typedefinable over $A$. 
(ii) Let $p \subseteq q$ be complete types over $A \subseteq B$, respectively. Then $q$ is a non-dividing extension of $p$ if and only if $D(p, \Xi)=D(q, \Xi)$ if and only if $D(q, \Xi)$ contains a maximal element of $D(p, \Xi)$.

(iii) Let $p \in \mathrm{S}(A)$. Then the property $p(x) \wedge x \downarrow_{A} y$ is type-definable in $x, y$.

In the terminology of [Ben02], we would say that complete types have definable independence.

Proof. (i) Using the independence theorem, $a \equiv_{A}^{\mathrm{Ls}} b \Leftrightarrow d_{A}(a, b) \leq 2$, and the latter is type-definable by thickness.

(ii) We have already seen in the proof of Theorem 1.15 that if $q$ contains a maximal element of $p$ then it cannot divide over $A$. Conversely, if $q$ does not divide over $A$ and $\xi_{<\alpha} \in D(p, \Xi)$, then we prove that $\xi_{<\alpha} \in D(q, \Xi)$ by induction on $\alpha$, as is done for local $D$-ranks in [KP97].

(iii) Let $\bar{\xi} \in D(p, \Xi)$ be maximal. Then $\operatorname{div}_{\bar{\xi}, A y}(x) \wedge p(x)$ defines the required property. $\mathbf{- 1 . 1 6}$

1.3. Groups. Let $\langle G, \cdot\rangle$ be a group definable over $\emptyset$ in a thick simple cat. Define the $G$-stratified total rank:

Definition 1.17. Let $p(x)$ be a partial type over $A, p(x) \vdash x \in G$. Let $\bar{\xi} \in \Xi^{\alpha}$. For $\alpha=0$ or limit we define $\bar{\xi} \in D_{G}(p, \Xi)$ as for $D(-, \Xi)$. If $\alpha=$ $\beta+1$, write $\xi_{\beta}=(\varphi, \psi)$. Then $\xi_{<\alpha} \in D_{G}(p, \Xi)$ if there are $b$ and $g \in G$ such that $\varphi(x, b)$ divides over $A$ with respect to $\psi$ and $\xi_{<\beta} \in D_{G}(p \wedge \varphi(g \cdot x, b), \Xi)$.

And similarly:

Definition 1.18. Let $A$ be some set, and $\bar{\xi}=\left(\left(\varphi_{i}, \psi_{i}\right): i<\alpha\right) \in \Xi^{\alpha}$. We say that $h$ satisfies $\operatorname{div}_{\bar{\xi}, A}^{G}$ if there exists a sequence $\left(b_{i}, g_{i}: i<\alpha\right)$ such that $\varphi_{i}\left(x, b_{i}\right)$ divides over $A \cup b_{>i} \cup g_{>i}$ with respect to $\psi_{i}$ for every $i<\omega$ and $\vDash \bigwedge_{i} \varphi_{i}\left(g_{i} \cdot h, b_{i}\right)$.

Adapting proofs from [Pil98] or [Wag01] we obtain:

FACT 1.19. (i) $D_{G}(-, \Xi) \subseteq \Xi^{<|T|^{+}}$.

(ii) $\bar{\xi} \in D_{G}(g / A, \Xi) \Leftrightarrow g \vDash \operatorname{div}_{\bar{\xi}, A}^{G}$.

(iii) $D_{G}(-, \Xi)$ is translation-invariant: $D_{G}(p(x), \Xi)=D_{G}(p(g \cdot x), \Xi)$.

(iv) $D_{G}(-, \Xi)$ witnesses dividing: if $p \subseteq q$ are complete types of elements of $G$, then $q$ is a non-dividing extension of $p$ if and only if $D_{G}(p, \Xi)=$ $D_{G}(q, \Xi)$ if and only if $D_{G}(q, \Xi)$ contains a maximal element of $D_{G}(p, \Xi)$.

Fix a set of parameters $A$. Let $\bar{\xi} \in D_{G}(G, \Xi)$ be maximal, and let $g \vDash$ $\operatorname{div}_{\bar{\xi}, A}^{G}$. If $h \in G, h \downarrow_{A} g$, then

$$
\begin{aligned}
\bar{\xi} \in D_{G}(g / A, \Xi) & =D_{G}(g / A h, \Xi)=D_{G}(h \cdot g / A h, \Xi) \\
& \subseteq D_{G}(h \cdot g, \Xi) \subseteq D_{G}(G, \Xi) .
\end{aligned}
$$


Since $\bar{\xi}$ is maximal in $D_{G}(G, \Xi)$ it is maximal in $D_{G}(h \cdot g, \Xi)$, whereby $A h \downarrow h \cdot g$. In other words, $g$ is right-generic.

Now let $\bar{\zeta} \in D_{G}(G, \Xi)$. Then we can find $h \in G$ such that $h \downarrow_{A} g$ and $\bar{\zeta} \in D_{G}(h / A, \Xi)$, whereby $h \downarrow_{A} h \cdot g$ and

$$
\begin{aligned}
\bar{\zeta} \in D_{G}(h / A, \Xi) & =D_{G}(h / A h \cdot g, \Xi)=D_{G}\left((h \cdot g)^{-1} \cdot h / A h \cdot g, \Xi\right) \\
& \subseteq D_{G}\left(g^{-1} / A, \Xi\right) .
\end{aligned}
$$

In other words, $D_{G}\left(g^{-1} / A, \Xi\right)=D_{G}(G, \Xi)$, so $g^{-1}$ is right-generic as well, and we conclude that $g$ is generic (that is, both right- and left-generic) and $D_{G}(g / A, \Xi)=D_{G}(G, \Xi)$.

We conclude:

FACT 1.20. $G$ contains generic elements over every set. For a set $A$ and $g \in G$, the following are equivalent:

(i) $g$ is generic over $A$.

(ii) $D_{G}(g / A, \Xi)=D_{G}(G, \Xi)$.

(iii) $D_{G}(g / A, \Xi)$ contains a maximal element of $D_{G}(G, \Xi)$.

So the generic elements of $G$ form a non-empty type-definable set.

REMARK 1.21. We could have also taken a path closer to [Pil98], proving that an element of $G$ whose sequence of $D_{G}\left(-, \varphi_{i}, \psi_{i}\right)$-ranks is maximal in some lexicographic order is generic.

We can show that for the existence of generics, the thickness assumption is not redundant. This example will be referred to as the White Queen's Paradox:

First, it was shown in [Ben03, Example 2.39, Remark 2.42] that there is a language for the category $\mathscr{H}$ of Hilbert spaces in which they are semiHausdorff, so in particular thick.

Alternatively, we gave in [Ben03, Example 2.39] a language for the category $\mathscr{B}$ of Banach spaces where addition, as well as multiplication by any scalar, are definable. If we took $\mathscr{H}$ with the same language the same proof would go through, whence the existence of a language for Hilbert spaces where addition is definable.

However, these two languages are incompatible:

Proposition 1.22. There is no language for $\mathscr{H}$ such that:

(i) Compactness holds.

(ii) Thickness holds.

(iii) Addition is type-definable.

Proof 1. Just adapt the proof of [Ben03, Remark 2.41]. - 1.22

Proof 2. We know that $\mathscr{H}$ is stable, and that independence coincides with orthogonality. Therefore, if such a language existed, then there would 
be generic elements for the additive groups. However, it is well known that these do not exist: if $a \downarrow b$ and both $a, b \neq 0$ then necessarily $a\left\lfloor a+b . \mathbf{m}_{1.22}\right.$

It would seem that the right approach in this case is to opt for thickness, and let go of the definable group. This opinion is strengthened by other phenomena concerning independence in $\mathscr{H}$, and in particular its triviality (two sets are independent if any two singletons are), which do not coincide with what we would expect to see in the presence of a group.

The reader who still finds it impossible to accept that a definable group that seems to be there is not, may wish to take the White Queen's advice from [Car82, Chapter V].

2. Categories of type-space functors. Let us recall from [Ben03] the definition of a type-space functor of a theory $T$. First, to every set $I$ (of which we think as a set of indices) it associates the topological space $\mathrm{S}_{I}(T)$. Then, to every mapping between sets $f: I \rightarrow J$, it associates the closed mapping $f^{*}: \mathrm{S}_{J}(T) \rightarrow \mathrm{S}_{I}(T)$, defined as $\operatorname{tp}\left(a_{j}: j \in J\right) \mapsto \operatorname{tp}\left(a_{f(i)}: i \in I\right)$. This defines a contravariant functor $\mathrm{S}(T): \mathcal{S}^{\text {op }} \rightarrow \mathcal{T}$, where $\mathcal{S}$ is the category of sets and $\mathcal{T}$ that of compact $T_{1}$ topological spaces with closed mappings as morphisms. We call $\mathrm{S}(T)$ the type-space functor of $T$.

We characterised in [Ben03, Definition 2.18 and Theorem 2.23] when an arbitrary functor $S: \mathcal{S}^{\mathrm{op}} \rightarrow \mathcal{T}$ is a type-space functor, i.e., isomorphic to $\mathrm{S}(T)$ for some $T$, by direct properties of $S$. Such an abstract type-space functor contains all the semantics of a theory while forgetting such unimportant information as its syntax. In particular, if $S \cong \mathrm{S}(T)$, then one can reconstruct $T$ from $S$ (up to Morleyisation). This renders type-space functors mathematical objects with equal status to that of positive Robinson theories, rather than mere features thereof.

Once we have observed all this we find that although positive Robinson theories seem to be anti-social creatures who live more or less on their own, the idea to use continuous maps between type-space functors as a means to express relations they may have is indeed very tempting.

The first such notion we consider is that of descriptions, the type-space functor analogue of the classical notion of interpretation.

About plain continuous maps between type-space functors we do not say much. However, if we restrict the theories under consideration to simple ones, and the notion of morphism to those that preserve independence, we obtain a more interesting notion. In particular, this gives a strong version of the stable dividing conjecture for which we know no counterexample.

2.1. Descriptions. We want to describe one theory in terms of another. When thinking in terms of type-space functors, a description should correspond to one type-space functor being "smaller" than the other. The general 
form of an object of a category being smaller than another is being a quotient of a sub-object, and we need to adapt this to our context.

We define:

Definition 2.1. (i) Let $X$ and $X^{\prime}$ be topological spaces, and $g$ : $X \rightarrow X^{\prime}$ a partial map. We say that $g$ is continuous if $g^{-1}(F) \subseteq X$ is closed whenever $F \subseteq X^{\prime}$ is closed (equivalently: if $\operatorname{dom}(g) \subseteq X$ is closed, and the total map $g: \operatorname{dom}(g) \rightarrow X^{\prime}$ is continuous in the induced topology).

(ii) Consider the following diagram, where $g$ and $h$ are partial maps and $f$ and $f^{\prime}$ are ordinary total maps:

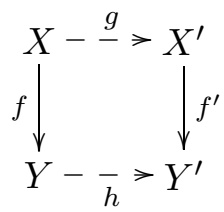

We say that the diagram commutes if whenever $x \in \operatorname{dom}(g)$ then $f(x) \in$ $\operatorname{dom}(h)$ and $h(f(x))=f^{\prime}\left(g(x)\right.$ ) (in short: $x^{\prime}=g(x) \Rightarrow f^{\prime}\left(x^{\prime}\right)=h(f(x))$ ).

(iii) Let $S, S^{\prime}$ be topological type-space functors. Then a partial continuous map $\mathfrak{d}: S \rightarrow S^{\prime}$ is given by a partial continuous map $\mathfrak{d}_{I}: S_{I} \rightarrow S_{I}^{\prime}$ for every $I$, such that for any $I, J$ and map $f: I \rightarrow J$ the following diagram commutes:

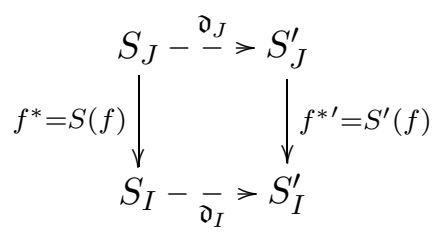

Thus, one way that a type-space functor $S^{\prime}$ can be "smaller" than another $S$ is for there to be a partial surjective continuous map $\mathfrak{d}: S \rightarrow S^{\prime}$. Let us fix one such partial map and try to understand what it means.

First, $\operatorname{dom}(\mathfrak{d})$ is a closed sub-functor of $S$, that is to say, $\operatorname{dom}\left(\mathfrak{d}_{I}\right) \subseteq S_{I}$ is a closed set for every set of indices $I$, and $f^{*}\left(\operatorname{dom}\left(\mathfrak{d}_{J}\right)\right) \subseteq \operatorname{dom}\left(\mathfrak{d}_{I}\right)$ for every map $f: I \rightarrow J$; this is a consequence of the assumption that $\mathfrak{d}$ and $f^{*}$ commute. In other words, given a tuple in a model of $S$ that realises a type in $\operatorname{dom}(\mathfrak{d})$ then every tuple obtained by changing the order, removing some elements, or duplicating some elements, also satisfies a type in dom $(\mathfrak{d})$. Thus, having a type in $\operatorname{dom}(\mathfrak{d})$ is a property of a set, rather than of a tuple, and it passes to subsets. Call such a set good (or $\mathfrak{d}$-good).

On the other hand, since $\operatorname{dom}(\mathfrak{d})$ is closed, the property of an $I$-tuple being an enumeration of a good set, possibly with repetitions, is definable by a partial type which will be denoted by $\delta_{I}$.

Lemma 2.2. A set is $\mathfrak{d}$-good if and only if all its finite subsets are. 
Proof. Let $a_{<\beta}$ enumerate a set. If it is good, we know that every subset is as well. Conversely, assume that every finite subset is good, that is to say, $\vDash \delta_{|w|}\left(a_{\in w}\right)$ for every finite $w \subseteq \beta$. Since an enumeration with repetitions of a good set satisfies $\delta$, we see that for every finite $w \subseteq \alpha$ there is a sequence $a_{<\alpha}^{w} \vDash \delta_{\alpha}$ such that $a_{i}^{w}=a_{i}$ for every $i \in w$. We conclude that $a_{<\alpha} \vDash \delta_{\alpha} \cdot \mathbf{m}_{2.2}$

The partial map $\mathfrak{d}: S \rightarrow S^{\prime}$ gives us a semantic translation of typedefinable properties from $S^{\prime}$ to $S$ : if $R \subseteq S_{n}^{\prime}$ is closed, then $\widetilde{R}=\mathfrak{d}_{n}^{-1}(R) \subseteq S_{n}$ is closed as well, by definition.

On the other hand, we also want to have a notion of syntactic translation from $S^{\prime}$ to $S$, and in order to have this we need a language. So fix a basis of closed sets for every $S_{n}^{\prime}$, and let $\mathcal{L}^{\prime}$ be the corresponding language, that is, the language consisting of an $n$-ary predicate $R\left(x_{<n}\right)$ for every basic closed set $R \subseteq S_{n}^{\prime}$. Let $\Delta^{\prime}$ be the positive fragment generated by these predicates, and let $\Sigma^{\prime}=\Sigma\left(\Delta^{\prime}\right)$ be the closure of $\Delta^{\prime}$ for existential quantification. If $\varphi\left(x_{<n}\right) \in \Sigma^{\prime}$ is an $n$-ary formula, we identify it with the closed set it defines in $S_{n}^{\prime}$, and let $\widetilde{\varphi}\left(x_{<n}\right)$ denote the partial type defining $\mathfrak{d}_{n}^{-1}(\varphi) \subseteq S_{n}$. On the other hand, we define $\widehat{\varphi}\left(x_{<n}\right)$ as the partial type obtained from $\varphi$ syntactically by replacing each occurrence of a predicate $R \in \mathcal{L}^{\prime}$ with $\widetilde{R}$ (so in particular, for $R \in \mathcal{L}^{\prime}, \widehat{R}=\widetilde{R}$ ).

Claim. Let $\varphi\left(x_{<n}\right) \in \Delta^{\prime}$ be an n-ary formula. Then $\widehat{\varphi} \wedge \delta_{n}$ is equivalent to $\widetilde{\varphi}$.

Proof. By induction on the syntactic structure of $\varphi$ :

- $\varphi=R$ is a predicate: by definition of $\widetilde{R}$.

- $\varphi \wedge \psi, \varphi \vee \psi$ : (positive) boolean combinations commute with inverse images.

- $\varphi\left(x_{<n}\right)=\psi\left(x_{f(0)}, \ldots, x_{f(m-1)}\right)$, where $\psi \in \Delta^{\prime}$ is an $m$-ary formula, and $f: m \rightarrow n$ is a map. In this case we say that $\varphi$ is obtained from $\psi$ by change of variables through $f$, and we also write $\varphi=f_{*}(\psi)=f^{*-1}(\psi)$. Then

$$
\begin{aligned}
\mathfrak{d}_{n}^{-1}(\varphi) & =\mathfrak{d}_{n}^{-1}\left(f^{*-1}(\psi)\right)=f^{*-1}\left(\mathfrak{d}_{m}^{-1}(\psi)\right) \wedge \delta_{n} \\
& =f^{*-1}\left(\widehat{\psi} \wedge \delta_{m}\right) \wedge \delta_{n}=f^{*-1}(\widehat{\psi}) \wedge f^{*-1}\left(\delta_{m}\right) \wedge \delta_{n} \\
& =f^{*-1}(\widehat{\psi}) \wedge \delta_{n}=\widehat{f_{*}(\psi)} \wedge \delta_{n}=\widehat{\varphi} \wedge \delta_{n},
\end{aligned}
$$

where $\mathfrak{d}_{n}^{-1}\left(f^{*-1}(\psi)\right)=f^{*-1}\left(\mathfrak{d}_{m}^{-1}(\psi)\right) \wedge \delta_{n}$ since $\mathfrak{d}$ and $f^{*}$ commute, $f^{*-1}\left(\delta_{m}\right)$ is implied by $\delta_{n}$ so it can be omitted, and $f_{*}(\widehat{\psi})=\widehat{f_{*}(\psi)}$ is a purely syntactic observation. $\boldsymbol{m}_{2.2}$

However, this is not sufficient: after all, existential quantification is also an important semantic construct, so we are going to add another requirement on $\mathfrak{d}$, that the above observation hold for every $\varphi \in \Sigma^{\prime}$, that is, when $\varphi$ is obtained from a $\Delta^{\prime}$-formula through existential quantification. 
One last twist is that, as with the classical notion of interpretation, we wish singletons of $S^{\prime}$ to be described by tuples of $S$.

This discussion leads us to the following definition:

Definition 2.3. (i) Let $\alpha$ be an ordinal, and $f: a \rightarrow b$ a map between sets. Then $f \times \alpha: a \times \alpha \rightarrow b \times \alpha$ is defined as $(x, i) \mapsto(f(x), i)$. If $S$ is a typespace functor, then $S_{\times \alpha}$ is the type-space functor defined by $\left(S_{\times \alpha}\right)_{a}=S_{a \times \alpha}$, and $S_{\times \alpha}(f)=S(f \times \alpha)$.

(ii) Let $S$ and $S^{\prime}$ be compact type-space functors, $\alpha_{\mathfrak{d}}$ an ordinal, and $\mathfrak{d}: S_{\times \alpha_{\mathcal{\jmath}}} \rightarrow S^{\prime}$ a surjective continuous partial map. Let $\mathcal{L}^{\prime}$ be the language corresponding to some basis of closed sets for the topology on $S^{\prime}$, and let $\Delta^{\prime}, \Sigma^{\prime}=\Sigma\left(\Delta^{\prime}\right)$ and $\varphi \mapsto \widetilde{\varphi}, \varphi \mapsto \widehat{\varphi}$ be as above. If in addition, for every $n$-ary formula $\varphi \in \Sigma^{\prime}, \widetilde{\varphi}$ is equivalent to $\widehat{\varphi} \wedge \delta_{n}$ (in other words, if $p \vdash \delta_{n}$ entails $\left.p \vdash \widehat{\varphi} \Leftrightarrow \mathfrak{d}_{n}(p) \vdash \varphi\right)$, then the pair $\left(\mathfrak{d}, \alpha_{\mathfrak{d}}\right)$ is a description of $S^{\prime}$ in $S$, written $\mathfrak{d}: S \rightarrow S^{\prime}$.

REMARK 2.4. We leave it to the reader to verify that:

(i) The definition of a description does not depend on the particular basis chosen for $S^{\prime}$.

(ii) The composition of two descriptions is a description: we obtain the description category of type-space functors.

REMARK 2.5. Let $\mathfrak{d}: S \rightarrow S^{\prime}$ be a description, so in particular $x \cong y$ is the property in $S$ defined by $\mathfrak{d}_{2}^{-1}(x=y)$. Then on any given good set, $\cong$ is an equivalence relation and moreover a congruence relation for all properties of the form $\widetilde{\varphi}$ (or $\widehat{\varphi}$ ). However, the union of two good sets is not necessarily good, so we cannot simply work in a hyperimaginary sort modulo $\cong$.

Proposition 2.6. An interpretation of one (first order) theory in another is a description.

Proof. Let $T$ and $T^{\prime}$ be first order theories. An interpretation of $T^{\prime}$ in $T$ is given by:

- a formula $X(\bar{x})$,

- a definable equivalence relation $E(\bar{x}, \bar{y})$ on the realisations of $X$,

- for each $n$-ary predicate $P \in \mathcal{L}^{\prime}$ (the language of $T^{\prime}$ ), an $E$-invariant formula $\widetilde{P}\left(\bar{x}_{<n}\right)$,

such that $T^{\prime}$ is precisely the theory of $X / E$, with each predicate $P$ being interpreted by $\widetilde{P}$. Moreover, we may assume that the interpretation uses parameters from a set $A$, in which case we write $X_{A}(\bar{x}), E_{A}(\bar{x}, \bar{y})$ and $\widetilde{P}_{A}\left(\bar{x}_{<n}\right)$. As we wish to work without parameters, we forget about $A$ and only retain $q(Z)=\operatorname{tp}(A)$.

Set $\delta_{\beta}\left(\left(\bar{x}_{i}, Z_{i}\right)_{i<\beta}\right)=q\left(Z_{0}\right) \wedge \bigwedge_{i<\beta}\left[Z_{i}=Z_{0} \wedge X_{Z_{0}}\left(\bar{x}_{i}\right)\right]$. Assume that $M \vDash \delta_{\beta}\left(\left(\bar{a}_{i}, A_{i}\right)_{i<\beta}\right)$, where $M$ is a model of $T$. Then we may write $A_{i}=A$ for 
all $i, X_{A}(M) / E_{A} \vDash T^{\prime}$, and we may define in this case $\mathfrak{d}_{\beta}\left(\operatorname{tp}^{M}\left(\left(\bar{a}_{i}, A\right)_{i<\beta}\right)\right)=$

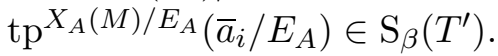

Write $\alpha_{\mathfrak{d}}=|\bar{x}|+|Z|$. Then $\operatorname{dom}\left(\mathfrak{d}_{\beta}\right)$ is defined in $\mathrm{S}_{\beta \times \alpha_{\mathfrak{d}}}(T)$ by $\delta_{\beta}$, and $\left(\mathfrak{d}, \alpha_{\mathfrak{d}}\right): T \rightarrow T^{\prime}$ is a description. We leave the verification of the details to the reader. $\mathbf{m} .6$

In the definition of a description, the only topological requirement was that the map $\mathfrak{d}: S \rightarrow S^{\prime}$ be continuous. But then $S^{\prime}$ has a maximal topology in which $\mathfrak{d}$ is continuous, and in this topology $S^{\prime}$ is a compact type-space functor (one easily verifies that all spaces are $T_{1}$ and compact, and all maps are continuous and closed). This makes sense: the maximal topology corresponds to the maximal language of $S^{\prime}$ that $S$ can describe.

Definition 2.7. Let $\mathfrak{d}: S \rightarrow S^{\prime}$ be a description.

(i) $\mathfrak{d}$ is optimal if $S^{\prime}$ has the maximal topology in which $\mathfrak{d}$ is continuous (in other words, $R \subseteq S_{n}^{\prime}$ is closed if and only if $\widetilde{R}=\mathfrak{d}_{n}^{-1}(R) \subseteq S_{n \times \alpha_{f}}$ is).

(ii) $\mathfrak{d}$ is closed if every $\mathfrak{d}_{n}$ is, that is to say, $\mathfrak{d}_{n}(R) \subseteq S_{n}^{\prime}$ is closed whenever $R \subseteq \operatorname{dom}\left(\mathfrak{d}_{n}\right)$ is.

Proposition 2.8. Let $\mathfrak{d}: S \rightarrow S^{\prime}$ be a description.

(i) If $\mathfrak{d}$ is closed, then it is optimal.

(ii) If $\mathfrak{d}$ is optimal, and $S^{\prime}$ is semi-Hausdorff, then $\mathfrak{d}$ is closed.

(iii) If $S^{\prime}$ is Hausdorff, then $\mathfrak{d}$ is closed.

(iv) If $\mathfrak{d}$ is closed and $S$ is Hausdorff, semi-Hausdorff or thick, then so is $S^{\prime}$.

Proof. (i) If $R \subseteq S_{n}^{\prime}$ and $\mathfrak{d}_{n}^{-1}(R)$ is closed, then $R=\mathfrak{d}_{n}\left(\mathfrak{d}_{n}^{-1}(R)\right)$ is closed as well.

(ii) We assume that $S^{\prime}$ is semi-Hausdorff and that $f$ is optimal, and we may assume as usual that $\alpha_{\mathfrak{d}}=1$. Consider $\cong=\mathfrak{d}_{2 n}^{-1}(\equiv) \subseteq S_{2 n}$, where $\equiv$ denotes equality of types; since $S^{\prime}$ is semi-Hausdorff, $\equiv$ is closed, and so is $\cong$. Thus, if $R \subseteq S_{n}$ is closed, then so is $R^{\prime}=\left\{p \in S_{n}: \exists q \in R q \cong p\right\}$, as it is defined by the property $R^{\prime}(x)=\exists y R(y) \wedge y \cong x$. On the other hand, $R^{\prime}=\mathfrak{d}_{n}^{-1}\left(\mathfrak{d}_{n}(R)\right)$, so by optimality $\mathfrak{d}_{n}(R)$ is closed.

(iii) A continuous map from a compact to a Hausdorff space is closed.

(iv) For semi-Hausdorff and thick this is easy: just take the image under $\mathfrak{d}$ of the closed set corresponding to $x \equiv y$ or to $d(x, y) \leq 1$, as may be the case.

For Hausdorff, assume that $p_{i} \in S_{n}^{\prime}$ for $i<2$ and $p_{0} \neq p_{1}$. Since $S_{n}$ is Hausdorff and each of $\mathfrak{d}_{n}^{-1}\left(p_{i}\right)$ is closed, there are closed $R_{i} \subseteq S_{n}$ such that $\mathfrak{d}_{n}^{-1}\left(p_{i}\right) \cap R_{i}=\emptyset$ for $i<2$ and $R_{0} \cup R_{1}=S_{n}$. Thus $p_{i} \notin \mathfrak{d}_{n}\left(R_{i}\right)$ and $S_{n}^{\prime}=\mathfrak{d}_{n}\left(R_{0}\right) \cup \mathfrak{d}_{n}\left(R_{1}\right)$; since moreover each $\mathfrak{d}_{n}\left(R_{i}\right)$ is closed, we obtain the required separation. $\mathbf{m}_{2.8}$ 
More interesting is the preservation of independence-related model-theoretic notions.

Lemma 2.9. Let $\mathfrak{d}: S \rightarrow S^{\prime}$ be a description, and $\Delta^{\prime}$ a positive fragment for $S^{\prime}$, and $\varphi(x, y), \psi(x, y), \chi\left(y_{<k}\right) \in \Delta^{\prime}$. If $\varphi$ and $\psi$ are contradictory then so are $\widetilde{\varphi}(\bar{x}, \bar{y})$ and $\widetilde{\psi}(\bar{x}, \bar{y})$; and if $\chi$ is a $k$-inconsistency witness for $\varphi$, then $\widetilde{\chi}\left(\bar{y}_{<k}\right)$ is a k-inconsistency witness for $\widetilde{\varphi}(\bar{x}, \bar{y})$.

Proof. For $\varphi$ and $\psi$ being contradictory, this is clear. If $\chi\left(y_{<y}\right)$ is a $k$ inconsistency witness for $\varphi(x, y)$, consider $\Upsilon\left(y_{<k}\right)=\exists x \chi\left(y_{<k}\right) \wedge \bigwedge_{i<k} \varphi\left(x, y_{i}\right)$ and $\widehat{\Upsilon}\left(y_{<k}\right)=\exists \bar{x} \widetilde{\chi}\left(\bar{y}_{<k}\right) \wedge \bigwedge_{i<k} \widetilde{\varphi}\left(\bar{x}, \bar{y}_{i}\right)$. Since $\tilde{\chi} \vdash \delta_{k}$, by the definition of a description $\widehat{\Upsilon}$ is equivalent to $\widetilde{\Upsilon}$. But as $\chi$ is a $k$-inconsistency witness for $\varphi$, $\Upsilon$ is contradictory, whereby $\widetilde{\Upsilon}$ is contradictory, and finally so is $\widehat{\Upsilon}$; thus $\tilde{\chi}$ is a $k$-inconsistency witness for $\widetilde{\varphi} \cdot 2.9$

Theorem 2.10. Let $S^{\prime}$ be described in $S$. If $S$ is stable or simple, then so is $S^{\prime}$.

Proof. Assume that $S^{\prime}$ is not stable. Then there are contradictory formulas $\varphi(x, y), \psi(x, y)$ such that $R(x=x, \varphi, \psi, 2)=\infty$ (see [Benb, Definition 2.1]). This is witnessed by a tree in a model of $S^{\prime}$, and if we pull back its type to $S$ we get a witness that $R(\bar{x}=\bar{x}, \widetilde{\varphi}, \widetilde{\psi}, 2)=\infty$, and $S$ is unstable.

Similarly, if $S^{\prime}$ is non-simple, there is a formula $\varphi$ and a $k$-inconsistency witness $\psi$ for $\varphi$ such that $D(x=x, \varphi, \psi)=\infty$, and again by pulling back the type of a witnessing tree we see that $D(\bar{x}=\bar{x}, \widetilde{\varphi}, \widetilde{\psi})=\infty$, and $S$ is not simple either. $\mathbf{m}_{2.10}$

REMARK 2.11. The assumption that we deal with a description, rather than merely a surjective continuous partial map, was not used in the stable case, as the inverse images of two disjoint sets are always disjoint. However, for the simple case, the assumption is not redundant. Consider, for example, that the type-space functor of the triangle-free random graph is a sub-functor of that of the ordinary random graph: the latter is simple, whereas the former is not.

With the additional hypothesis of thickness, we can point out the precise difference between stability and simplicity:

Proposition 2.12. Let $T$ be a thick simple cat. Then it is unstable if and only if it describes the random graph.

Proof. One direction is clear. For the other, assume that $T$ is unstable. Then we can find an element $a$, and a set $A$ satisfying $\operatorname{bdd}(A)=\operatorname{dcl}(A)$ (see Proposition 3.9), so that every type over $A$ is a Lascar strong type, and yet $\operatorname{tp}(a / A)$ is non-stationary. Then we can find $a_{0}, a_{1} \equiv_{A} a$, and a finite tuple $b \downarrow_{A} a_{0} a_{1}$ such that $a_{0} \not \equiv_{A b} a_{1}$. 
Choose $b_{0}^{\prime} \equiv_{A} b$ such that $b_{0}^{\prime} \downarrow_{A} a_{0} b$, and take $p(x, Z)=\operatorname{tp}\left(a_{0} b_{0}^{\prime}, A\right)$. By the independence theorem, we may now find $a_{0}^{\prime} \downarrow_{A} a_{0} b b_{0}^{\prime}$ such that $p\left(a_{0}^{\prime} b, A\right)$ and $a_{0}^{\prime} b_{0}^{\prime} \equiv_{A} a_{0} b$. Similarly, we may find $b_{1}^{\prime} \downarrow_{A} a_{1} b$ such that $p\left(a_{1} b_{1}^{\prime}, A\right)$, and then $a_{1}^{\prime} \downarrow_{A} a_{1} b b_{1}^{\prime}$ such that $p\left(a_{1}^{\prime} b, A\right)$ and $a_{1}^{\prime} b_{1}^{\prime} \equiv_{A} a_{1} b$. Write

$$
\begin{aligned}
& r_{0}(x, y, Z)=\operatorname{tp}\left(a_{0} b_{0}^{\prime}, a_{0}^{\prime} b, A\right) \vee \operatorname{tp}\left(a_{0}^{\prime} b, a_{0} b_{0}^{\prime}, A\right), \\
& r_{1}(x, y, Z)=\operatorname{tp}\left(a_{1} b_{1}^{\prime}, a_{1}^{\prime} b, A\right) \vee \operatorname{tp}\left(a_{1}^{\prime} b, a_{1} b_{1}^{\prime}, A\right) .
\end{aligned}
$$

Then $r_{i}(x, y, A)$ are symmetric relations, implying $p(x, A), p(y, A)$ and $x \downarrow_{A} y$, and $r_{0}(x, y, Z) \wedge r_{1}(x, y, Z)$ is contradictory.

For $n<\omega$ write

$$
\begin{aligned}
\delta_{n}\left(\left(x_{i}, Z_{i}\right)_{i<n}\right)=\bigwedge_{i<\beta} Z_{i}= & Z_{0} \wedge p\left(x_{i}, Z_{0}\right) \\
& \wedge \bigwedge_{j<i} r_{0}\left(x_{i}, x_{j}, Z_{0}\right) \vee r_{1}\left(x_{i}, x_{j}, Z_{0}\right) \vee x_{i}=x_{j} \\
& \wedge \bigwedge_{w \subseteq n}\left[x_{i} \downarrow_{Z_{0}} x_{\in w} \vee \bigvee_{j \in w} x_{i}=x_{j}\right] .
\end{aligned}
$$

In particular, $x_{i} \downarrow_{Z_{0}} x_{\in w}$ is type-definable since $T$ is assumed to be thick and $\operatorname{tp}\left(x / Z_{0}\right)$ is known.

The partial type $\delta_{n}$ says first that all the $Z_{i}$ are equal to some $Z$, and then that the set $\left\{x_{i}: i<n\right\}$, when considered without repetitions, is $Z$-independent. Moreover if $x_{i} \neq x_{j}$ then they satisfy either $r_{0}$ or $r_{1}$, and we know they cannot satisfy both. For a possibly infinite ordinal $\beta$, define $\delta_{\beta}\left(\left(x_{i}, Z_{i}\right)_{i<\beta}\right)=\bigwedge_{n<\omega, w \in[\beta]^{n}} \delta_{n}\left(\left(x, Z_{i}\right)_{i \in w}\right)$. Then again $\delta_{\beta}$ says that all the $Z_{i}$ are the same and equal to some $Z$, and by the finite character of dividing the set $\left\{x_{i}: i<\beta\right\}$ is $Z$-independent when considered without repetitions. Let $T_{\mathrm{gr}}$ be the theory of the random graph. As a first order theory, $T_{\text {gr }}$ eliminates quantifiers in the language $\{=, R\}$, so we can consider it as a positive Robinson theory with a positive fragment $\Delta_{\mathrm{gr}}$ generated by $\{=, R, \not R\}$, where $x \not R y$ means $x \neq y \wedge \neg R(x, y))$. In particular, the type of a tuple of vertices is determined by its $\Delta_{\mathrm{gr}}$-type: interpreting $r_{0}$ as an edge and $r_{1}$ as a non-edge we obtain a unique partial map $\mathfrak{d}: \mathrm{S}_{\times \alpha_{\mathfrak{d}}}(T) \rightarrow \mathrm{S}\left(T_{\mathrm{gr}}\right)$ with $\operatorname{dom}(\mathfrak{d})$ being defined by $\delta, \alpha_{\mathfrak{d}}=$ $|x|+|Z|$, and $\widetilde{R}\left(x, Z, x^{\prime}, Z^{\prime}\right)=\left[Z=Z^{\prime} \wedge r_{0}\left(x, x^{\prime}, Z\right)\right], \widetilde{R}\left(x, Z, x^{\prime}, Z^{\prime}\right)=$

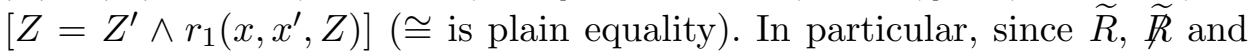
equality are type-definable, $\mathfrak{d}$ is continuous. That the maps $\mathfrak{d}_{\beta}$ commute with the restriction maps $f^{*}$ (of $\mathrm{S}_{\times \alpha_{0}}(T)$ and of $\mathrm{S}\left(T_{\mathrm{gr}}\right)$ ) is an easy exercise.

Assume that $p\left(\left(x_{i}, Z_{i}\right)_{i<n}\right)=\operatorname{tp}\left(\left(c_{i}, A_{i}\right)_{i<n}\right) \vdash \delta_{n}$. Then all the $A_{i}$ are equal, so write them as $A$, and write $p^{\prime}\left(x_{<n}\right)=\mathfrak{d}_{n}(p)$. If $\varphi\left(x_{<n+m}, y_{<m}\right)$ $\in \Delta_{\mathrm{gr}}$ and $\psi\left(x_{<n}\right)=\exists x_{n \leq i<m} \varphi\left(x_{<n+m}\right) \in \Sigma_{\mathrm{gr}}$, we want to prove that $p \vdash \widehat{\psi} \Leftrightarrow p^{\prime} \vdash \psi$. We may write $\varphi$ as a disjunction of conjunctions of basic predicates, and since disjunction commutes with existential quantification 
we may deal with each of the disjuncts separately, and assume that $\varphi$ is a conjunction of basic predicates.

For $\Leftarrow$, we may assume that $m=1$, since the general case would follow by induction. If $\varphi \vdash x_{i}=x_{n}$ for some $i$, just take $c_{n}=c_{i}$. Otherwise, as all types over $A$ are Lascar strong, and the set of distinct elements in $\left\{c_{i}: i<n\right\}$ is $A$-independent, successive applications of the independence theorem yield $c_{n}$ satisfying $r_{0}\left(c_{n}, c_{i}, A\right)$ or $r_{1}\left(c_{n}, c_{i}, A\right)$ for all $i<n$ such that $\varphi \vDash x_{i} R x_{n}$ or $\varphi \vDash x_{i} \not R x_{n}$, respectively.

For $\Rightarrow$, assume that $p^{\prime} \forall \psi$, that is to say, $p^{\prime}\left(x_{<n}\right) \wedge \varphi\left(x_{<n+m}\right)$ is contradictory. Since both $p^{\prime}$ and $\varphi$ (by assumption) can be written as conjunctions of basic predicates, $p^{\prime}\left(x_{<n}\right) \wedge \varphi\left(x_{<n+m}\right)$ is also such a conjunction, and its being contradictory means that there are $i<j<m+n$ such that $x_{i}=x_{j} \wedge x_{i} R x_{j}, x_{i}=x_{j} \wedge x_{i} \not R x_{j}$ or $x_{i} R x_{j} \wedge x_{i} \not R x_{j}$ appear in this conjunction, which would make $p \wedge \widehat{\varphi}$ contradictory as well.

Finally, the argument for $\Leftarrow$ also shows that $\mathfrak{d}$ is surjective, and we conclude that $\mathfrak{d}: T \rightarrow T_{\text {gr }}$ is a description. $\mathbf{m}_{2.12}$

A much more interesting example of a description is given in [Bena], where we prove that if $T$ is a thick simple cat and $T^{\mathfrak{P}}$ is the theory of its lovely pairs, then $T^{\mathfrak{P}}$ has a closed description in $T$. For example, we can use Proposition 2.8 to prove that such properties as being Hausdorff, semi-Hausdorff, or thick pass to $T^{\mathfrak{P}}$ (the proofs actually appearing in [Bena] are merely special cases of the argument given above). This also gives an alternative proof of the simplicity of $T^{\mathfrak{P}}$, using Theorem 2.10 .

2.2. Stable representations. We saw above that the notion of a map between type-space functors can be a useful tool for the expression of relations between two theories. Given a simple theory, we may consider it interesting to describe not its language, but rather its notion of independence, in another simple theory, and with some luck maybe even in a stable one. Doing so, we may allow some of the power of expression of the language to be lost on the way: indeed, if we want to represent the notion of independence of an unstable simple theory in a stable one, the definable sets that cause the instability will have to go.

Put in more concrete terms, the map will know how to go the other way round (always from the theory with more definable sets to the one with less); it also has to be total and to preserve independence. If it is in addition surjective, we obtain some sort of reduct; but we allow it now to be, in which case the term representation may seem more adequate.

Definition 2.13. (i) Let $S$ and $S^{\prime}$ be simple, and $\mathfrak{r}: S \rightarrow S^{\prime}$ a continuous map of topological functors. Assume that for every triplet of tuples of elements $(A, B, C)$ from a model of $S$, and for every such triplet 
$\left(A^{\prime}, B^{\prime}, C^{\prime}\right)$ from $S^{\prime}$ such that $\operatorname{tp}\left(A^{\prime}, B^{\prime}, C^{\prime}\right)=\mathfrak{r}(\operatorname{tp}(A, B, C))$, we have: $A \downarrow_{C} B \Leftrightarrow A^{\prime} \downarrow_{C^{\prime}} B^{\prime}$. Then $f$ preserves independence.

(ii) A simple type-space functor $S$ has a stable representation if there is a stable $S^{\prime}$ and an independence preserving map $\mathfrak{r}: S \rightarrow S^{\prime}$.

Having a stable representation is a strong condition. In particular, it implies stable dividing:

Recall that a partial type $\pi(x, y)$ is stable if there is no indiscernible sequence $\left(a_{i} b_{i}: i<\omega\right)$ such that $\vDash \pi\left(a_{i}, b_{j}\right) \Leftrightarrow i \leq j$. This is equivalent to: there is no formula $\psi$ contradicting $\pi$ such that $R(x=x, \pi, \psi, 2)=\infty$.

Proposition 2.14. If $S$ is thick, simple, and has a stable representation then it has stable dividing, meaning that if $a \mathbb{\bigsqcup}_{c} b$ divides over $c$ there is a stable partial type $\pi(x, y z)$ such that $\vDash \pi(a, b c)$, and $\pi(x, b c)$ divides over $b$. If $S$ has a stable representation in a first order theory (or even a Robinson theory) then $\pi$ can be taken to be a formula (that is to say, $\neg \pi$ is also a positive property).

Proof. Let $\mathfrak{r}: S \rightarrow S^{\prime}$ be a stable representation. Assume that $a \not_{c} b$ in $S$, and write $p(x, y z)=\operatorname{tp}(a, b, c), p^{\prime}(x, y z)=\mathfrak{r}(p)$. If $\vDash p^{\prime}\left(a^{\prime}, b^{\prime} c^{\prime}\right)$, then $a^{\prime} \mathbb{Z}_{c^{\prime}} b^{\prime}$ by assumption, so there is a formula $\varphi(x, y z) \in p^{\prime}$ such that $\varphi\left(x, b^{\prime} c^{\prime}\right)$ divides over $c^{\prime}$ (all this happens in $\left.S^{\prime}\right)$. In $S$, take a Morley sequence $\left(b_{i}: i<\omega\right)$ over $c$ containing $b$, and let $\left(b_{i}^{\prime}: i<\omega\right)$ be a sequence in $S^{\prime}$ such that $a^{\prime} b^{\prime} c^{\prime} \bar{b}^{\prime} \vDash \mathfrak{r}(\operatorname{tp}(a b c \bar{b}))$. Then $\left(b_{i}^{\prime}: i<\omega\right)$ is a Morley sequence over $c^{\prime}$ containing $b$, so $\left\{\varphi\left(x, b_{i}^{\prime} c^{\prime}\right)\right\}$ is inconsistent. Set $\widetilde{\varphi}=\mathfrak{r}^{-1}(\varphi)$. Then $\widetilde{\varphi}$ is a stable partial type in $S$ (it cannot order an indiscernible sequence, or else $\varphi$ would order one in $\left.S^{\prime}\right)$, and $\left\{\widetilde{\varphi}\left(x, b_{i} c\right)\right\}$ is inconsistent. Then $\widetilde{\varphi}(x, b c)$ is a stable witness to $a \bigotimes_{c} b$.

If $S^{\prime}$ is totally disconnected, then $\varphi$ defines a clopen set, whereby $\widetilde{\varphi}$ is clopen as well.

EXAMPLE 2.15. Let PSF be the theory of pseudo-finite fields, ACF the theory of algebraically closed fields. Let $\mathfrak{r}: \mathrm{S}(\mathrm{PSF}) \rightarrow \mathrm{S}(\mathrm{ACF})$ be defined by sending each type to its quantifier-free part. Then $\mathfrak{r}$ is surjective and preserves independence. Thus PSF has a stable representation, although it does not have a stable reduct in the classical sense.

EXAMPLE 2.16. Similarly, let ACFA be the theory of algebraically closed fields with a generic automorphism. Let $\mathrm{ACF}_{\times \omega}$ be the theory of $\omega$-tuples of elements of $\mathrm{ACF}$ (defined by $\mathrm{S}\left(\mathrm{ACF}_{\times \omega}\right)=\mathrm{S}_{\times \omega}(\mathrm{ACF})$ ). $\mathrm{ACF}_{\times \omega}$ has totally disconnected type-spaces, but $=$ is not clopen (that is, $\neq$ is not a positive relation). Then we have a natural map $\mathrm{S}(\mathrm{ACFA}) \rightarrow \mathrm{S}_{\times \omega}(\mathrm{ACF})$, which consists in sending the type of a tuple to the quantifier free type of its orbit. This map preserves independence, and ACFA has a stable representation. 
EXAMPLE 2.17. More generally, let $T$ be a stable first order theory, and let $T_{A}$ be the theory $T$ with a generic automorphism constructed in [Pil00]; it is proved there essentially that $T_{A}$ is a simple cat. In fact, one can show that if $T$ is a first order theory with the PAPA, and in particular if $T$ is stable, then $T_{A}$ is a Robinson theory in the sense of [Hru97] in a properly chosen language. Similarly, let $T_{\mathrm{fix}}$ be the simple Robinson theory of the fixed set in $T_{A}$ (i.e., the analogue of PSF). The same stable representations as above exist for $T_{A}$ and $T_{\text {fix }}$ (this is actually a restatement of the characterisation of independence in $T_{A}$ ).

3. A few remarks. This section consists of a collection of useful remarks, concerning cats and simplicity. Throughout, $T$ is an arbitrary cat.

3.1. On basic hyperimaginaries. The elimination of arbitrary hyperimaginaries in favour of small, or basic, hyperimaginaries was first suggested (as far as we know) in [BPW01] for first order theories. The same holds in cats, the sole difference being that the bound for the size is $|T|$ rather than $\omega$.

Definition 3.1. A hyperimaginary sort $x / E$ is basic if $|x| \leq|T|$. A hyperimaginary is basic if it is in a basic sort.

Lemma 3.2. Let $E(x, y)$ be a definable equivalence relation. Then for every $\varphi(x, y) \in E$ there is an equivalence relation $E_{\varphi}$ such that $\varphi \in E_{\varphi} \subseteq E$ and $\left|E_{\varphi}\right| \leq|T|$.

Proof. For every $\psi \in E$, we know that $E(x, y) \wedge E(y, z) \vdash \psi(x, z)$. This means that $E(x, y) \wedge E(y, z) \wedge \chi(x, y)$ is contradictory for every $\chi(x, y)$ contradicting $\psi$, and finitely many formulae from $E$ suffice for this. If $\psi$ is written only with the variables actually appearing in it, it has at most $|T|$ negations. Therefore there is $E_{\psi}^{\prime} \subseteq E$ such that $E_{\psi}^{\prime}(x, y) \wedge E_{\psi}^{\prime}(y, z) \vdash \psi(x, z)$ and $\left|E_{\psi}^{\prime}\right| \leq|T|$. Define

$$
\begin{aligned}
E_{\varphi}^{0}(x, y) & =\{\varphi(x, y)\}, \\
E_{\varphi}^{n+1}(x, y) & =E_{\varphi}^{n}(x, y) \cup E_{\varphi}^{n}(y, x) \cup \bigcup_{\psi \in E_{\varphi}^{n}} E_{\psi}^{\prime}(x, y)
\end{aligned}
$$

and $E_{\varphi}=\bigcup_{n<\omega} E_{\varphi}^{n}$. Then $E_{\varphi}$ will do. -3.2

COROLlary 3.3. Every hyperimaginary is interdefinable with a set of basic hyperimaginaries.

Proof. Let $a / E$ be given, and let $A=\left\{a / E_{\varphi}: \varphi \in E\right\}$. Then clearly every $a / E_{\varphi}$ is definable over $a / E$. Conversely, if $E_{\varphi}(a, b)$ for every $\varphi \in E$, then in particular $\varphi(a, b)$ for every $\varphi \in E$ and therefore $E(a, b)$, which shows that $a / E$ is definable over $A$. 
Since for every $\varphi \in E$ there are at most $|T|$ variables actually appearing in $E_{\varphi}(x, y)$, we may replace each $a / E_{\varphi}$ with an interdefinable basic hyperimaginary. $\mathbf{m} .3$

3.2. Boundedly closed models. We wish to show that models are boundedly closed. We have the semi-Hausdorff case, which turns out to be a mere rephrasing of the first order results, and the general case, which requires us to replace "e.c. model" by " $|T|^{+}$-compact model".

Definition 3.4. A set $A$ is boundedly closed if $a \in \operatorname{bdd}(A) \Rightarrow a \in \operatorname{dcl}(A)$ for every hyperimaginary $a$.

FACT 3.5. A set $A$ is boundedly closed if and only if $a \in \operatorname{bdd}(A) \Rightarrow a \in$ $\operatorname{dcl}(A)$ for every basic hyperimaginary a.

We recall that $\varphi(x, y)$ is thin if $\bigwedge_{i<j<\omega} \varphi\left(x_{i}, x_{j}\right)$ is inconsistent, and:

FACT 3.6. Let $a, b$ be two tuples, and $A$ a set. Then $a, b, \ldots$ begin an $A$-indiscernible sequence if and only if $p(x, y)=\operatorname{tp}(a, b / A)$ contains no thin formula (with parameters in A).

Proof. Left to right is clear. For right to left, it suffices to observe that if $\bigwedge_{i<j<\lambda} p\left(x_{i}, x_{j}\right)$ is inconsistent (for any infinite $\lambda$ ) this is due to some thin formula in $p$.

Lemma 3.7. Let $M$ be a $\kappa^{+}$-compact model of $T, A$ be a subset of $M$ and $a$ be a tuple possibly outside $M$ such that $|a|+|A|+|T| \leq \kappa$. Then there is $c \in M$ such that $d_{A}(a, c) \leq 1$ ( $a, c, \ldots$ begin an $A$-indiscernible sequence).

Proof. Let $\left\{\varphi_{i}(x, y): i<\kappa\right\}$ enumerate all the thin formulas with parameters in $A$, where $x, y$ are in the sort of $a$. Recall that a set $t \subseteq \bigcup_{\alpha \in \text { Ord }} \kappa^{\alpha}$ is a tree if it is closed for initial segments, and we write $\sigma<\eta$ to say that $\sigma$ is a proper initial segment of $\eta$. To such a tree associate the partial type

$$
\Phi_{t}\left(x_{\in t}\right)=\bigwedge_{\sigma<\eta \in t} \varphi_{\eta(|\sigma|)}\left(x_{\eta}, x_{\sigma}\right)
$$

We claim that if $t$ contains a branch of length $\kappa^{+}$, then $\Phi_{t}$ is inconsistent. Indeed, if $\eta$ is such a branch, then there is some $i<\kappa$ such that $\eta(\alpha)=i$ for infinitely many $\alpha<\kappa^{+}$, which would make $\Phi_{t}$ inconsistent as $\varphi_{i}$ is thin. This means in particular that if $\Phi_{t}$ is consistent then $t \subseteq \kappa^{<\kappa^{+}}$, and by compactness and Zorn's lemma the set $\left\{t: \Phi_{t}\right.$ is consistent $\}$ contains a maximal element. Let $t$ denote from now on such a maximal tree.

For $\eta \in t$, assume that we have $\left(a_{\sigma}: \sigma<\eta\right) \subseteq M$ such that $\Phi_{t} \wedge \bigwedge_{\sigma<\eta} x_{\sigma}=a_{\sigma}$ is consistent. Then $\Psi(y)=\exists\left(x_{\eta}: \eta \in t\right) \Phi_{t} \wedge \bigwedge_{\sigma<\eta} x_{\sigma}=$ $a_{\sigma} \wedge x_{\eta}=y$ is consistent, and has at most $\kappa$ parameters, so it is realised by some $a_{\eta} \in M$, and now $\Phi_{t} \wedge \bigwedge_{\sigma \leq \eta} x_{\sigma}=a_{\sigma}$ is consistent. Proceeding like this we obtain $\left(a_{\eta}: \eta \in t\right)$ realising $\Phi_{t}$ in $M$. 
Define a sequence of ordinals $i_{\alpha}<\kappa$ by induction on $\alpha<\kappa^{+}$, such that, writing $\eta_{\alpha}=\left(i_{\beta}: \beta<\alpha\right)$, we have $\eta_{\alpha} \in t$ and $\varphi_{i_{\alpha}}\left(a, a_{\eta_{\alpha}}\right)$ for every $\alpha$. Assuming that $\eta_{\alpha}$ is already constructed, we observe that necessarily $\eta_{\alpha} \in t$ : if not, define $t^{\prime}=t \cup\left\{\eta_{\alpha}\right\}$, so realising $x_{\eta_{\alpha}}$ by $a$ we obtain a contradiction to the maximality of $t$. If there is $i<\kappa$ such that $\vDash \varphi_{i}\left(a, a_{\eta_{\alpha}}\right)$ then we define $i_{\alpha}=i$ and continue the construction. If not, then we define $c=a_{\eta_{\alpha}}$, and the construction stops.

As the construction must stop at some point, we get $c \in M$ such that the pair $a, c$ satisfies no thin formula over $A$, so $d_{A}(a, c)=1$. $\mathbf{m}_{3.7}$

Lemma 3.8. Let a/E be a hyperimaginary, and say that $a / E \in \operatorname{bdd}(A)$. Then there is $B \subseteq A$ with $|B| \leq|a|+|T|$ such that $a / E \in \operatorname{bdd}(B)$.

Proof. Write $p(x)=\operatorname{tp}(a / A)$. For every formula $\varphi(x, y)$ which contradicts $E(x, y)$, we know that $\bigwedge_{i<j<\omega} p\left(x_{i}\right) \wedge \varphi\left(x_{i}, x_{j}\right)$ is inconsistent since $a / E \in \operatorname{bdd}(A)$, so there is a formula $\psi_{\varphi}\left(x, b_{\varphi}\right) \in p$ such that $\bigwedge_{i<j<\omega} \psi_{\varphi}\left(x_{i}, b_{\varphi}\right)$ $\wedge \varphi\left(x_{i}, x_{j}\right)$ is inconsistent. Let $B$ be the set of all such parameters $b_{\varphi}$, of which there are at most $|A|+|T|$. Then $a / E \in \operatorname{bdd}(B)$. $\mathbf{m}_{3.8}$

Hence:

Proposition 3.9. Every $|T|^{+}$-compact model is boundedly closed.

Proof. Let $M$ be $|T|^{+}$-compact. We need to show that if $a / E \in \operatorname{bdd}(M)$ then $a / E \in \operatorname{dcl}(M)$, and it would suffice to show this for the case where $a / E$ is a basic hyperimaginary. Since $a / E$ is basic, $|a| \leq|T|$, so there is $A \subseteq M$ with $|A| \leq|T|$ such that $a / E \in \operatorname{bdd}(A)$. Then by Lemma 3.7 there exists $c \in M$ such that $d_{A}(a, c) \leq 1$. Thus $d_{A}(a / E, c / E) \leq 1$, and as $a / E \in \operatorname{bdd}(A)$ we conclude that $a / E=c / E \in \operatorname{dcl}(M) . \quad$ 3.9

REMARK 3.10. The brute-force approach to the proof of Lemma 3.7 would have been to try to find a maximal colouring $c:[\alpha]^{2} \rightarrow \kappa$, where $\alpha$ is an ordinal and $\bigwedge_{i<\alpha} \wedge \bigwedge_{j<i} \varphi_{c(\{i, j\})}\left(x_{i}, x_{j}\right)$ is consistent. Then we see that necessarily $|\alpha| \leq 2^{\kappa}$ : if not, then by Erdős-Rado we obtain an infinite homogeneous subset $I \subseteq \alpha$ which gives a contradiction. With this bound, we would need a $2^{\kappa}$-compact model.

Similarly, we could have tried to prove Proposition 3.9 by enumerating all the $A$-conjugates of $a / E$ and then realising them all in $M$; again, we would need $2^{|T|}$-compactness.

However, upon closer inspection, one sees that the proof of the ErdösRado theorem does not consider the entire coloured graph, but rather a sub-graph which is in fact a tree, for whose realisation $\kappa^{+}$-compactness (or $|T|^{+}$-compactness in the particular case used for Proposition 3.9) suffices, whence the refined result. 
Using the semi-Hausdorff hypothesis, we can get stronger results, while only assuming that the model is e.c.:

Lemma 3.11. Assume that $T$ is semi-Hausdorff. Then every type over an e.c. model has non-splitting extension to any set.

Proof. Let $M \in \mathcal{M}, p \in \mathrm{S}(M)$, and $A \supseteq M$. Let $q_{0}(x)=p(x) \cup$ $\left\{a \equiv_{x} b: n<\omega, a, b \in A^{n}, a \equiv_{M} b\right\}$. For any $\varphi(x, m) \in p$ there is $c \in M$ such that $\varphi(c, m)$, and $a \equiv_{M} b$ implies in particular $a \equiv_{c} b$. Thus $q_{0}$ is finitely consistent and therefore consistent. Any completion to a complete type over $A$ would be non-splitting, and we are done. 3.11

REMARK 3.12. In a Hausdorff theory, we can also prove this using a suitable notion of co-heir.

Proposition 3.13. In a semi-Hausdorff theory:

(i) Equality of Lascar strong type over $A$ is the transitive closure of having the same type over an e.c. model containing A.

(ii) Every e.c. model is boundedly closed.

Proof. (i) We prove as in [KP97]: The equivalence relation we described is clearly bounded, so it is implied by equality of Lascar strong types. For the converse, assume that $M \in \mathcal{M}$ and $\operatorname{tp}(a / M)=\operatorname{tp}(b / M)=p$, and let $N$ and $q$ be as above. Let $c_{i} \in N$ realise $q \uparrow_{M a b c_{<i}}$. Then $a, c_{0}, c_{1}, \ldots$ and $b, c_{0}, c_{1}, \ldots$ are both $M$-indiscernible sequences, so $a \equiv_{M}^{\mathrm{Ls}} c_{0} \equiv_{M}^{\mathrm{Ls}} b$.

(ii) Let $M \in \mathcal{M}$ and $a \in \operatorname{bdd}(M)$. Then $\operatorname{tp}(a / M) \vdash \operatorname{lstp}(a / M) \vdash \operatorname{tp}(a / a)$, whereby $a \in \operatorname{dcl}(M) \cdot \mathbf{m} 3.13$

REMARK 3.14. In a Hausdorff theory, we can also prove that e.c. models are boundedly closed directly, using a variant of the first order proof.

\subsection{On full simplicity}

Definition 3.15. A cat $T$ is fully simple if it is simple and every complete type over a set of real elements is extensible (that is, has non-dividing extensions to every set).

The problem with full simplicity is that it cannot be stated in terms of finiteness of $D$-ranks, and does not follow from stability. Another problem is that we do not know whether full simplicity implies that every type over a hyperimaginary domain is extensible; here we give an approximation of such a result, which should suffice for most practical purposes.

Proposition 3.16. Let $T$ be simple, and let $\mathcal{A}$ be the class of all hyperimaginaries a such that every type over a is extensible. Then the following 
are equivalent:

(i) $a \in \mathcal{A}$.

(ii) There is a real tuple $b$ such that $a \in \operatorname{dcl}(b)$ and $\operatorname{tp}(b / a)$ is extensible.

(iii) There are $b, c \in \mathcal{A}$ such that $a$ is interbounded with $\mathrm{Cb}(b / c)$.

(iv) There is a real tuple $b$ such that $a \in \operatorname{bdd}(b)$ and $\operatorname{tp}(b / a)$ is extensible. Moreover, if a is any tuple of hyperimaginaries having these properties, then a has it as well.

Proof. (i) $\Rightarrow$ (ii). Write $a=b_{E}$, that is, the $E$-class of a real tuple $b$. Then $a \in \operatorname{dcl}(b)$ and $\operatorname{tp}(b / a)$ is extensible.

(ii) $\Rightarrow$ (iii). Since $\operatorname{tp}(b / a)$ is extensible, we can find $c \equiv_{a} b$ such that $b \downarrow_{a} c$, and then $a$ is interbounded with $\mathrm{Cb}(b / c)$.

(iii) $\Rightarrow$ (iv). Since $b \in \mathcal{A}$, we can find a real tuple $b^{\prime}$ such that $b \in \operatorname{dcl}\left(b^{\prime}\right)$ and $b^{\prime} \downarrow_{b} c$. Then $b^{\prime} \downarrow_{\mathrm{Cb}(b / c)} c$, so $a$ is interbounded with $\mathrm{Cb}\left(b^{\prime} / c\right)$ as well. Let $\bar{b}^{\prime}$ be a Morley sequence for $b^{\prime}$ over $c$; then $\operatorname{Cb}\left(b^{\prime} / c\right) \in \operatorname{dcl}\left(\bar{b}^{\prime}\right)$, and $\operatorname{tp}\left(\bar{b}^{\prime} / \mathrm{Cb}\left(b^{\prime} / c\right)\right)$ is extensible. Therefore $a \in \operatorname{bdd}\left(\bar{b}^{\prime}\right)$ and $\operatorname{tp}\left(\bar{b}^{\prime} / a\right)$ is extensible.

(iv) $\Rightarrow(\mathrm{i})$. Assume that $a \in \operatorname{bdd}(\bar{b})$ and $\operatorname{tp}(\bar{b} / a)$ is extensible, and let $c$ be any tuple. Since $T$ is fully simple, $\operatorname{tp}(c / \bar{b})$ is extensible, and so is $\operatorname{tp}(c / a \bar{b})$. Since $\operatorname{tp}(\bar{b} / a)$ is also extensible, $\operatorname{tp}(\bar{b} c / a)$ is extensible, and in particular $\operatorname{tp}(c / a)$ is.

For the moreover part, write $\bar{a}=\left(a_{i}: i<\alpha\right)$ such that for every $i$ there is a real tuple $b_{i}$ such that $a_{i} \in \operatorname{dcl}\left(b_{i}\right)$ and $\operatorname{tp}\left(b_{i} / a_{i}\right)$ is extensible. We may take them such that $b_{i} \downarrow_{a_{i}} \bar{a} b_{<i}$. Then $\operatorname{tp}(\bar{b} / \bar{a})$ is extensible. $\mathbf{m}_{3.16}$

So as far as the only hyperimaginaries we ever wish to construct are canonical bases, we may assume that every type over a hyperimaginary is extensible.

\subsection{On the universal quantifier for hyperimaginary sorts}

Definition 3.17. A cat $T$ is open if for every $n, m<\omega$, the projection map $\mathrm{S}_{n+m}(T) \rightarrow \mathrm{S}_{n}(T)$ is open. In other words, the universal quantifier is positive. A cat $T$ is strongly open if for every map $f: m \rightarrow n$ (not necessarily injective), the map $f^{*}: \mathrm{S}_{n}(T) \rightarrow \mathrm{S}_{m}(T)$ is open. This is equivalent to: $T$ is open and inequality is positive.

Proposition 3.18. Let $T$ be an open cat, and $T^{\text {heq }}$ be obtained by adding all basic hyperimaginary sorts. Then $\mathrm{S}\left(T^{\mathrm{heq}}\right)$ is open.

Proof. We need to show that every map of the form $\mathrm{S}_{x / E, y / F}(T) \rightarrow$ $\mathrm{S}_{x / E}(T)$, where $x / E$ and $y / F$ are hyperimaginary sorts, is open. Consider a formula $\varphi(x, y)$ (of course, only finitely many variables actually appear 
in $\varphi)$. Then by definition, $\varphi(x / E, y / F)=\exists z w E(z, x) \wedge F(w, y) \wedge \varphi(z, w)$. We claim that $\forall y / F \varphi(x / E, y / F)$ is positive, that is, defines a closed set.

Indeed, consider the partial type $\pi(x)=\{\forall y \exists z w \chi(x, z) \wedge \varrho(y, w) \wedge$ $\varphi(z, w): \chi \in E, \varrho \in F\}$. Each formula of the form $\forall y \exists z w \chi(x, z) \wedge \varrho(y, w) \wedge$ $\varphi(z, w)$ has finitely many variables actually appearing in it, and by the assumption of openness it is equivalent to a positive partial type. Therefore $\pi$ is a partial type, and one sees that it is equivalent to $\forall y / F \varphi(x / E, y / F)$ : one implication is clear, the other by compactness.

Since it suffices to verify the openness of a map on a basis, we conclude that the map $\mathrm{S}_{x / E, y / F}(T) \rightarrow \mathrm{S}_{x / E}(T)$ is open, whereby $\forall$ is a positive quantifier, sending closed sets to closed sets. $\mathbf{B}_{3.18}$

We know that a first order theory is open (and in fact strongly open): in fact, a Robinson theory is first order if and only if it is open. Thus in particular, hyperimaginary sorts in a first order theory are open (but clearly not strongly open).

\section{References}

[Bena] I. Ben-Yaacov, Lovely pairs of models: the non-first order case, preprint.

[Benb] - Simplicity in compact abstract theories, preprint.

[Ben02] - Group configurations and germs in simple theories, J. Symbolic Logic 67 (2002), 1581-1600.

[Ben03] - Positive model theory and compact abstract theories, J. Math. Logic 3 (2003), 85-118.

[BPV] I. Ben-Yaacov, A. Pillay, and E. Vassiliev, Lovely pairs of models, Ann. Pure Appl. Logic, to appear.

[BPW01] S. Buechler, A. Pillay, and F. O. Wagner, Supersimple theories, J. Amer. Math. Soc. 14 (2001), 109-124.

[BTW] I. Ben-Yaacov, I. Tomašić, and F. O. Wagner, Constructing an almost hyperdefinable group, preprint.

[BW] I. Ben-Yaacov and F O. Wagner, On almost orthogonality in simple theories, J. Symbolic Logic, to appear.

[Car82] L. Carroll, Through the Looking-Glass and What Alice Found There, in: The Complete Illustrated Works of Lewis Carroll, ed. by Edward Guiliano, Avenel Books, New York, 1982, 81-176.

[CLPZ01] E. Casanovas, D. Lascar, A. Pillay, and M. Ziegler, Galois groups of first order theories, J. Math. Logic 1 (2001), 305-319.

[Hru97] E. Hrushovski, Simplicity and the Lascar group, preprint, 1997.

[KP97] B. Kim and A. Pillay, Simple theories, Ann. Pure Appl. Logic 88 (1997), 149-164.

[Pil98] A. Pillay, Definability and definable groups in simple theories, J. Symbolic Logic 63 (1998), 788-796.

[Pil00] - Forking in the category of existentially closed structures, in: Connections between Model Theory and Algebraic and Analytic Geometry, A. Macintyre (ed.), Quad. Mat. 6, Aracne, Roma, 2000, 23-42. 
[Wag01] F. O. Wagner, Groups in simple theories, in: Proc. Logic Colloq. 2001, to appear.

Department of Mathematics

Massachusetts Institute of Technology

77 Massachusetts Avenue, Room 2-101

Cambridge, MA 02139-4307, U.S.A.

E-mail: pezz@math.mit.edu

URL: http://www-math.mit.edu/ pezz

Received 24 October 2002;

in revised form 9 October 2003 